\title{
Management of symptomatic radiation necrosis after stereotactic radiosurgery and clinical factors for treatment
}

\section{response}

\author{
Mutlay Sayan ${ }^{1}$, Teuta Zoto Mustafayev², Aykut Balmuk ${ }^{3}$ Swati Mamidanna', Erva Seyma Sare Kefelioglu4, \\ Gorkem Gungor ${ }^{4}$, Anupama Chundury', Nisha Ohri', Ercan Karaarslan ${ }^{5}$, Enis Ozyar ${ }^{3}$, Banu Atalar ${ }^{3}$ \\ ${ }^{1}$ Department of Radiation Oncology, Rutgers Cancer Institute of New Jersey, New Brunswick, NJ, USA \\ ${ }^{2}$ Department of Radiation Oncology, Acibadem Maslak Hospital, Istanbul, Turkey \\ ${ }^{3}$ School of Medicine, Mehmet Ali Aydinlar Acibadem University, Istanbul, Turkey \\ ${ }^{4}$ Institute of Health Sciences, Mehmet Ali Aydinlar Acibadem University, Istanbul, Turkey \\ ${ }^{5}$ Department of Radiology, School of Medicine, Mehmet Ali Aydinlar Acibadem University, Istanbul, Turkey
}

Received: March 25, 2020

Revised: June 26, 2020

Accepted: July 6, 2020

\section{Correspondence:}

Mutlay Sayan

Department of Radiation Oncology,

Rutgers Cancer Institute of New

Jersey, 195 Little Albany Street, New

Brunswick, NJ 08901, USA.

Tel: +1-732-235-3939

Fax: +1-732-253-3953

E-mail:ms2641@cinj.rutgers.edu

ORCID:

https://orcid.org/0000-0002-0101-0951
Purpose: Approximately $10 \%$ of patients who received brain stereotactic radiosurgery (SRS) develop symptomatic radiation necrosis (RN). We sought to determine the effectiveness of treatment options for symptomatic RN, based on patient-reported outcomes.

Materials and Methods: We conducted a retrospective review of 217 patients with 414 brain metastases treated with SRS from 2009 to 2018 at our institution. Symptomatic RN was determined by appearance on serial magnetic resonance images (MRIs), MR spectroscopy, requirement of therapy, and development of new neurological complaints without evidence of disease progression. Therapeutic interventions for symptomatic RN included corticosteroids, bevacizumab and/or surgical resection. Patient-reported therapeutic outcomes were graded as complete response (CR), partial response (PR), and no response.

Results: Twenty-six patients experienced symptomatic RN after treatment of 50 separate lesions. The mean prescription dose was 22 Gy (range, 15 to $30 \mathrm{~Gy}$ ) in 1 to 5 fractions (median, 1 fraction). Of the 12 patients managed with corticosteroids, 6 patients (50\%) reported CR and 4 patients (33\%) PR. Of the 6 patients managed with bevacizumab, 3 patients (50\%) reported CR and 1 patient (18\%) PR. Of the 8 patients treated with surgical resection, all reported CR (100\%). Other than surgical resection, age $\geq 54$ years (median, 54 years; range, 35 to 81 years) was associated with $C R$ (odds ratio $=8.40$; $95 \%$ confidence interval, 1.27-15.39; $p=0.027$ ).

Conclusion: Corticosteroids and bevacizumab are commonly utilized treatment modalities with excellent response rate. Our results suggest that patient's age is associated with response rate and could help guide treatment decisions for unresectable symptomatic RN.

Keywords: Brain, Radiosurgery, Necrosis

Copyright (C) 2020 The Korean Society for Radiation Oncology

This is an Open Access article distributed under the terms of the Creative Commons Attribution Non-Commercial License (http://creativecommons.org/licenses/by-nc/4.0/) which permits unrestricted non-commercial use, distribution, and reproduction in any medium, provided the original work is properly cited. 


\section{Introduction}

There has been an increase in the incidence of brain metastases over the years with an estimated 100,000 to 240,000 people diagnosed each year in the United States, almost 10 times greater than the incidence of primary brain tumors [1]. Increased incidence of brain metastases is believed to be secondary to an earlier detection through improved diagnostic techniques in addition to newer systemic therapies which allow patients to live longer with metastatic disease [2]. Despite these newer therapies/detection modalities, once the primary neoplasm has metastasized to the brain, despite multimodal treatment, the outcomes are usually poor [3].

The approach to management in these cases varies depending on multiple factors such as the site, type and staging of the primary tumor, the size and number of metastases along with the age and performance status of the individual. The main therapeutic options for brain metastases include surgical resection, whole brain radiation therapy (WBRT), stereotactic radiosurgery (SRS), and/or systemic therapy such as immunotherapy. SRS is slowly emerging as a popular therapeutic option due to its short and convenient treatment course, high rates of local controls, and its relative mitigation of neurocognitive deficits when compared to WBRT [4]. Despite the many benefits seen with SRS, it is not without adverse effects, including radiation necrosis (RN) a late toxicity usually observed months to years postSRS [5].

$\mathrm{RN}$ is an inflammatory reaction leading to irreversible necrotic degeneration of the brain tissue at the site of previous cerebral irradiation [6]. The management of RN depends on the symptomatic presentation which can vary from mild symptoms such as nausea or headaches to more severe symptoms such as cognitive or neurological deficits and seizures. While smaller, asymptomatic RN can be managed with observation and careful monitoring, larger more symptomatic RN must be treated definitively with various therapeutic options such as corticosteroids, bevacizumab, surgical resection, or some combination of above.

Corticosteroids act by modulating inflammatory changes and edema, often leading to rapid symptomatic improvement after initiation [7]. However, steroid toxicity and withdrawal pose dosing-related challenges and complications. Bevacizumab, a monoclonal antibody which inhibits vascular endothelial growth factor A (VEGF-A), hinders angiogenesis and has shown benefit in symptomatic RN cases [8]. Surgical resection of the necrosed tissue also effectively manages RN but also has its disadvantages as it is an invasive procedure [9]. Each of therapeutic modality has its own relative benefits and drawbacks. In this study, we have aimed to evaluate the effectiveness of these three therapeutic options in the management of symptomatic $\mathrm{RN}$, utilizing patient-reported outcomes.

\section{Materials and Methods}

We conducted an IRB-approved retrospective review of patients with brain metastases who were treated with SRS at Acibadem Maslak Hospital between 2009 to 2018 (No. 2019116). The study was conducted in accordance with the principles of the Declaration of Helsinki. All patients were treated with robotic linear accelerator (CyberKnife, Sunnyvale, CA, USA)-based SRS. Demographic, clinical, and brain lesion information was collected, including age, gender, location of brain metastases, number of brain metastases, tumor size, tumor volume, addition of WBRT, and prescription dose.

A post-SRS brain magnetic resonance imaging (MRI) was obtained 4 to 6 weeks after treatment followed by serial MRIs every 3 months thereafter. At each follow-up visit, RN was rated according to the Radiation Therapy Oncology Group (RTOG) CNS toxicity criteria with diagnosis being determined on serial MRIs, MR spectroscopy, and development of new CNS/neurologic complaints without evidence of disease progression [10].

The management of RN depends on the symptomatic presentation which can vary from mild symptoms such as headaches to more severe symptoms such as cognitive or neurological deficits and seizures. In this study, symptomatic RN was managed with corticosteroids alone, bevacizumab and/or surgical resection at the discretion of the treating physician. Patients who received bevacizumab and those who underwent surgical resection received a trial of corticosteroids first. Once diagnosed, patients were seen 2-4 weeks after treatment of symptomatic RN and then subsequently followed with appropriate diagnostic imaging and clinical follow-up every 3 months. At last follow-up visit, patients graded the RN treatment outcomes as complete response (CR), partial response (PR), and no response (NR). The primary endpoint was to determine the patient-reported response rate with treatment of symptomatic RN. Statistical analyses were performed using SPSS statistical software version 25 (IBM Corp., Armonk, NY, USA).

\section{Results}

We identified 414 brain metastases treated with SRS in 217 patients at our institution from 2009 to 2018. Twenty-six patients experienced symptomatic RN after treatment of 50 lesions (Table 1). Median follow-up was 10.1 months (range, 6.2 to 100.0 months) since SRS. Median number of RN per patient was 1 (range, 1 to 4). Median patient age was 54 years (range, 35 to 81 years). Most common histologies were primary non-small cell lung cancer (69\%) and breast cancer (23\%). Median tumor size was $0.77 \mathrm{~cm}$ (range, 0.01 to $1.96 \mathrm{~cm}$ ). The most common metastases location was parietal lobe (40\%), occipital lobe (30\%), and temporal lobes (18\%). The 
mean prescription dose was 22 Gy (range, 15 to $30 \mathrm{~Gy}$ ) in 1 to 5 fractions (median, 1 fraction). WBRT was received by $66 \%$ of the patients either before or after SRS. Among the patients with symptomatic RN, RTOG CNS grade 2 toxicity was observed in 19 patients (73\%) and grade 3 in 7 patients (27\%) (Table 2). The main symptoms were headache in 20 patients (77\%), muscle weakness in 3 patients (11\%), seizures in 2 patients (8\%), and cognitive impairment in 1 patient (4\%).

Patients with symptomatic RN lesions were managed by one of three treatment modalities namely oral corticosteroids $(n=12)$, bevacizumab ( $n=6)$ or surgical resection $(n=8)$. Median follow-up was 8.5 months (range, 3.2 to 96.0 months) since treatment of symptomatic RN. A total of 17 patients (66\%) reported CR, 5 patients (19\%) reported $P R$, and 4 patients (15\%) reported NR. Among the 12 patients managed with corticosteroids, 6 patients (50\%) reported CR, 4 patients (33\%) PR, and 2 patients (17\%) reported NR. Among the 6 patients managed with bevacizumab, 3 patients (50\%) reported CR, 1 patient (18\%) reported $P R$, and 2 patients (33\%) reported NR. Among the 8 patients treated with surgical resection, all reported CR (100\%). Other than surgery, factor associated with $\mathrm{CR}$ on univariate logistic regression was age

Table 1. Demographic and clinical characteristics of the patients included in the study $(n=26)$

\begin{tabular}{lc}
\hline Characteristic & Value \\
\hline Number of brain lesion & 50 \\
Age (yr) & $54(35-81)$ \\
Gender & \\
Female & $16(62)$ \\
Male & $10(38)$ \\
Histology & \\
NSCLC & $18(69)$ \\
Breast & $6(23)$ \\
Melanoma & $1(4)$ \\
Parotid & $1(4)$ \\
Location & \\
Frontal & $6(12)$ \\
Parietal & $20(40)$ \\
Temporal & $9(18)$ \\
Occipital & $15(30)$ \\
Radiation therapy & $17(66)$ \\
WBRT & \\
SRS & $22^{\text {a) }}(15-30)$ \\
Dose (Gy) & $1(1-5)$ \\
Number of fractions &
\end{tabular}

Values are presented as median (range) or number (\%).

NSCLC, non-small cell lung cancer; WBRT, whole brain radiation therapy; SRS, stereotactic radiosurgery.

a) measured mean. $\geq 54$ years (median, 54 years; range, 35 to 81 years; odds ratio = 8.40; 95\% confidence interval, 1.27-15.39; $p=0.027$ ) (Table 3).

\section{Discussion and Conclusion}

SRS has emerged as an important therapy in the management of brain metastases and over the years has evolved into its own therapeutic option as opposed to adjunctive treatment after WBRT [1113]. The goal of SRS is to achieve a more effective therapeutic response by administering a higher dose of radiation per fraction while reducing the integral dose to normal brain parenchyma. The utilization of SRS is dictated by the clinical scenario in question and it can be employed in a variety of forms such as a planned boost post-WBRT, a conformally administered adjuvant treatment after surgical resection, and/or as single-modality treatment as well [14].

Though SRS is considered an effective treatment option for limited brain metastases with decreased neurocognitive deficits compared to WBRT, it is not without its own toxicities. RN is one such

Table 2. Incidence of CNS toxicities in patients with symptomatic radiation necrosis

\begin{tabular}{lc}
\hline Variable & Value \\
\hline Type of complication & $20(77)$ \\
Headache & $3(11)$ \\
Motor deficits & $2(8)$ \\
Seizure & $1(4)$ \\
Cognitive deficits & \\
RTOG CNS toxicity & 0 \\
Grade 0 & 0 \\
Grade 1 & $19(73)$ \\
Grade 2 & $7(27)$ \\
Grade 3 & 0 \\
Grade 4 &
\end{tabular}

Values are presented as number (\%).

CNS, central nervous system; RTOG, Radiation Therapy Oncology Group.

Table 3. Factors associated with complete response on univariate logistic regression

\begin{tabular}{lccc}
\hline Variable & OR & $95 \% \mathrm{Cl}$ & $\mathrm{p}$-value \\
\hline Gender (male vs. female) & 1.140 & $0.22-5.84$ & 0.873 \\
Age $\geq 54$ years & 8.400 & $1.27-15.4$ & 0.027 \\
Non-NSCLC primary & 0.414 & $0.08-2.18$ & 0.298 \\
RTOG CNS toxicity (grade 2 vs. & 0.720 & $0.42-2.75$ & 0.746 \\
grade 3) & & & \\
WBRT & 0.141 & $0.14-1.38$ & 0.093 \\
\hline
\end{tabular}

$\mathrm{OR}$, odds ratio; $\mathrm{Cl}$, confidence interval; $\mathrm{NSCLC}$, non-small cell lung cancer; RTOG, Radiation Therapy Oncology Group; CNS, central nervous system; WBRT, whole brain radiation therapy. 
rare but serious long-term effect of SRS, occurring in around 5\%$25 \%$ of patients who have been treated with SRS [15]. It usually presents as a late complication 6 to 18 months after SRS and can manifest in a variety of presentations including incidental detection on routine imaging to seizures, headaches, and/or with focal neurologic deficits. History of prior radiation exposure, dose of radiation administered, volume of brain parenchyma irradiated, concurrent systemic therapy and intrinsic radio-sensitivity of the primary histology are all risk factors in the development of RN [16]. The primary underlying pathophysiologic mechanism for the development of necrosis is believed to be due to radiation-induced injury of the cerebral blood vessels which leads to secondary brain parenchymal damage. Another theory suggests direct radiation-induced damage to glial cells resulting in white matter demyelination and necrosis [17].

The treatment options for RN are often determined based its clinical presentation of symptoms. Asymptomatic RN is typically managed with observation with close clinical and radiological monitoring, whereas symptomatic RN often necessitates active treatment $[18,19]$. Corticosteroids, humanized monoclonal antibodies against VEGF, anticoagulants, hyperbaric oxygen therapy, and surgical resection are some of the available treatment options for the management of RN.

Corticosteroids have long been considered the first line treatment option for symptomatic RN, with several trials demonstrating their benefit. They provide benefit in patients with RN by reducing inflammatory changes and decreasing the permeability of the blood-brain barrier [7]. The adverse effects associated with longterm steroid use such as gastric ulceration, steroid-induced myopathy, iatrogenic Cushing syndrome, and steroid toxicity are major drawbacks associated with this treatment [20]. Another option which has generated significant interest in the management of RN is bevacizumab, which is a monoclonal antibody against VEGF. The upregulation of vascular inflammatory changes, which plays a crucial role in the pathophysiology of $\mathrm{RN}$, can be modulated by administration of this drug $[8,21]$. A randomized placebo-controlled double-blind trial conducted by Levin et al. [22] presented Class I evidence for bevacizumab as a treatment option for RN. The administration of bevacizumab has been tempered due to some of the adverse effects of this drug, such as thrombosis, hemorrhage, and impaired wound healing.

In patients who are refractory to treatment with corticosteroids or bevacizumab, surgical resection can be considered as a treatment option. Surgical resection provides the benefit of prompt symptomatic relief by decreasing mass effect and relieving intracranial pressure, in addition to providing confirmation of diagnosis via histopathological evaluation. A retrospective study conducted by Telera et al. [23] concluded that surgical resection in a group of 15 patients with symptomatic RN provided symptomatic relief in 14 patients. The high risk of morbidity associated with surgical resection of the lesion, however, is often a deterrent in choosing this option. This potential risk of morbidity has been highlighted in a study conducted by McPherson et al. [9] which emphasized that surgical intervention should be reserved as an option only in treatment-refractory RN.

Limitations of our study include its small sample size, retrospective design, and inherent confounding factors that cannot be completely accounted for in a non-randomized study. In addition, patient-reported therapeutic outcomes were graded using a non-validated questionnaire which may be influenced by patient's background and desirability of answer.

Studies comparing three modalities of treatment of $\mathrm{RN}$, such as corticosteroids, bevacizumab and surgical resection are relatively rare. In our study, we have aimed to further shed light on this aspect and have concluded that corticosteroids and bevacizumab are commonly utilized treatment modalities in our institution with a $50 \%$ CR rate. Our results also indicate that the patient's age is associated with response rate and could help guide treatment decisions for unresectable symptomatic RN.

\section{Conflict of Interest}

No potential conflict of interest relevant to this article was reported.

\section{References}

1. Lowery FJ, Yu D. Brain metastasis: unique challenges and open opportunities. Biochim Biophys Acta Rev Cancer 2017;1867:4957.

2. Fink KR, Fink JR. Imaging of brain metastases. Surg Neurol Int 2013:4:S209-19.

3. Suh $\mathrm{JH}$. Stereotactic radiosurgery for the management of brain metastases. N Engl J Med 2010;362:1119-27.

4. Sittenfeld SM, Suh JH, Murphy ES, Yu JS, Chao ST. Contemporary management of 1-4 brain metastases. Front Oncol 2018;8:385.

5. Song YP, Colaco RJ. Radiation necrosis: a growing problem in a case of brain metastases following whole brain radiotherapy and stereotactic radiosurgery. Cureus 2018;10:e2037.

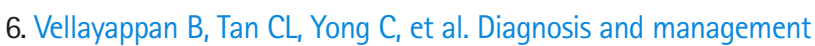
of radiation necrosis in patients with brain metastases. Front Oncol 2018;8:395.

7. Kotsarini C, Griffiths PD, Wilkinson ID, Hoggard N. A systematic review of the literature on the effects of dexamethasone on the 
brain from in vivo human-based studies: implications for physiological brain imaging of patients with intracranial tumors. Neurosurgery 2010;67:1799-815.

8. Kim JH, Chung YG, Kim CY, Kim HK, Lee HK. Upregulation of VEGF and FGF2 in normal rat brain after experimental intraoperative radiation therapy. J Korean Med Sci 2004;19:879-86.

9. McPherson CM, Warnick RE. Results of contemporary surgical management of radiation necrosis using frameless stereotaxis and intraoperative magnetic resonance imaging. J Neurooncol 2004;68:41-7.

10. Cox JD, Stetz J, Pajak TF. Toxicity criteria of the Radiation Therapy Oncology Group (RTOG) and the European Organization for Research and Treatment of Cancer (EORTC). Int J Radiat Oncol Biol Phys 1995;31:1341-6.

11. O'Beirn M, Benghiat $H$, Meade $S$, et al. The expanding role of radiosurgery for brain metastases. Medicines (Basel) 2018;5:90.

12. Shinde A, Akhavan D, Sedrak M, Glaser S, Amini A. Shifting paradigms: whole brain radiation therapy versus stereotactic radiosurgery for brain metastases. CNS Oncol 2019;8:CNS27.

13. Sayan M, Zoto Mustafayev T, Sahin B, et al. Evaluation of response to stereotactic radiosurgery in patients with radioresistant brain metastases. Radiat Oncol J 2019;37:265-70.

14. Schimmel WC, Gehring K, Eekers DB, Hanssens PE, Sitskoorn MM. Cognitive effects of stereotactic radiosurgery in adult patients with brain metastases: a systematic review. Adv Radiat Oncol 2018:3:568-81.

15. Kohutek ZA, Yamada Y, Chan TA, et al. Long-term risk of radionecrosis and imaging changes after stereotactic radiosurgery for brain metastases. J Neurooncol 2015;125:149-56.

16. Sneed PK, Mendez J, Vemer-van den Hoek JG, et al. Adverse radiation effect after stereotactic radiosurgery for brain metastases: incidence, time course, and risk factors. J Neurosurg 2015;123:373-86.

17. Miyatake S, Nonoguchi N, Furuse M, et al. Pathophysiology, diagnosis, and treatment of radiation necrosis in the brain. Neurol Med Chir (Tokyo) 2015;55:50-9.

18. Patel TR, McHugh BJ, Bi WL, Minja FJ, Knisely JP, Chiang VL. A comprehensive review of MR imaging changes following radiosurgery to 500 brain metastases. AJNR Am J Neuroradiol 2011;32:1885-92.

19. Wang $Y X$, King AD, Zhou $H$, et al. Evolution of radiation-induced brain injury: MR imaging-based study. Radiology 2010;254:2108.

20. Giglio P, Gilbert MR. Cerebral radiation necrosis. Neurologist 2003;9:180-8.

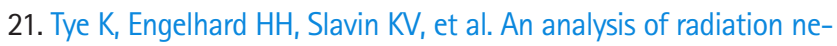
crosis of the central nervous system treated with bevacizumab. J Neurooncol 2014;117:321-7.

22. Levin VA, Bidaut $L$, Hou $P$, et al. Randomized double-blind placebo-controlled trial of bevacizumab therapy for radiation necrosis of the central nervous system. Int J Radiat Oncol Biol Phys 2011;79:1487-95.

23. Telera $S$, Fabi A, Pace $A$, et al. Radionecrosis induced by stereotactic radiosurgery of brain metastases: results of surgery and outcome of disease. J Neurooncol 2013;113:313-25. 